\title{
Correction of Sunken Upper Eyelids by Anchoring the Central Fat Pad to the Medial Fat Pad during Upper Blepharoplasty
}

\author{
Myeong Su Jeon, Gyu Yong Jung, Dong Lark Lee, Hea Kyeong Shin \\ Department of Plastic and Reconstructive Surgery, Dongguk University College of Medicine, Gyeongju, Korea
}

Background Many methods have been proposed for the correction of sunken upper eyelids. These methods include surgical treatments, such as micro-fat, dermofat, or fascia-fat grafts, or the use of alloplastic materials. Here, we present our experience of sunken upper eyelid correction involving the simple addition of anchoring the central fat pad to the medial fat pad during upper blepharoplasty.

Methods We performed 74 cases of upper blepharoplasty with sunken upper eyelid correction between October 2013 and September 2014. The lateral portion of the central fat pad was partially dissected to facilitate anchoring. The medial fat pad was gently exposed and then pulled out to facilitate anchoring. After the rotation of the dissected lateral portion of the central fat pad by $180^{\circ}$ to the medial side, it was anchored spreading to the medial fat pad. Photographs taken at 6 months postoperatively were presented to three physicians for objective assessment. Of the 74 patients, 54 patients followed at 6 months postoperatively were included in this retrospective, objective assessment.

Results Sunken eyelids were effectively corrected in 51 of the 54 patients, but 3 had minimal effect because preaponeurotic fat pads had been removed during previous upper blepharoplasty. In addition to correcting sunken eyelids, lateral bulging was corrected and a better definition of the lateral portion of upper lid creases was obtained.

Conclusions Anchoring the central fat pad to the medial fat pad provides an effective means of correcting sunken upper eyelids during upper blepharoplasty.

Keywords Blepharoplasty / Eyelids / Esthetics
Correspondence: Gyu Yong Jung Department of Plastic and Reconstructive Surgery, Dongguk University College of Medicine, 87 Dongdae-ro, Gyeongju 780-350, Korea

Tel: +82-54-770-8242

Fax: +82-54-770-8501

E-mail: jnjbeauty@naver.com
This article was presented as a free paper at the 72nd Congress of the Korean Society of Plastic and Reconstructive Surgeons on November 7-9, 2014, in Seoul, Korea.

No potential conflict of interest relevant to this article was reported.

Received: 17 Jan $2015 \bullet$ Revised: 8 Apr 2015 • Accepted: 10 Apr 2015

pISSN: 2234-6163・ elSSN: 2234-6171 • http://dx.doi.org/10.5999/aps.2015.42.4.469 • Arch Plast Surg 2015;42:469-474

\section{INTRODUCTION}

Upper blepharoplasty is one of the most popular aesthetic operations in Korea. People request upper blepharoplasty for different reasons, but many simply want to correct sunken upper eyelids because they give the impression of being old and tired. Sunken upper eyelids can occur from congenital or acquired causes, such as aging, complications of previous periocular surgery, or facial trauma [1], and several methods are available to correct the condition, such as surgical treatment, micro-fat grafting, dermofat grafting, fascia-fat grafting, or the use of alloplastic materials [1-11].

In this article, we present our experience of sunken upper eyelid correction by upper blepharoplasty with simple anchoring of 
the central fat pad to the medial fat pad.

\section{METHODS}

We performed 74 cases of upper blepharoplasty for correcting sunken upper eyelids between October 2013 and September 2014. Clinical photographs were taken at the front gaze with eyes closed and open before the operation. Postoperative photographs were taken at 3-month and 6-month postoperative visits.

Photographs taken at 6-month postoperative visits were objectively assessed by three physicians. Photographs were evaluated using a four-point system, where 0 , no correction; 1 , minimal correction; 2, moderate correction; and 3, full correction. Among the 74 patients, 54 patients who were followed up for 6 months postoperatively were included in this objective assessment. The evaluation scores of the three physicians were subjected to an intraclass correlation analysis. All the statistical analyses were performed using IBM SPSS ver. 19.0 (IBM Inc., Armonk, NY, USA).

\section{Technique}

With the patients in a supine position, we blocked the lacrimal, supratrochlear, and supraorbital nerves by administering $2 \%$ lidocaine/1:80,000 epinephrine; intravenous midazolam and ketamine were added for short-term sedation. Skin and orbicularis muscle were incised proceeding from the lateral to the medial position. After dissecting pretarsal skin and a muscle flap from the levator aponeurosis, the orbital septum was opened to expose the preaponeurotic space (Fig. 1). Central and medial fat

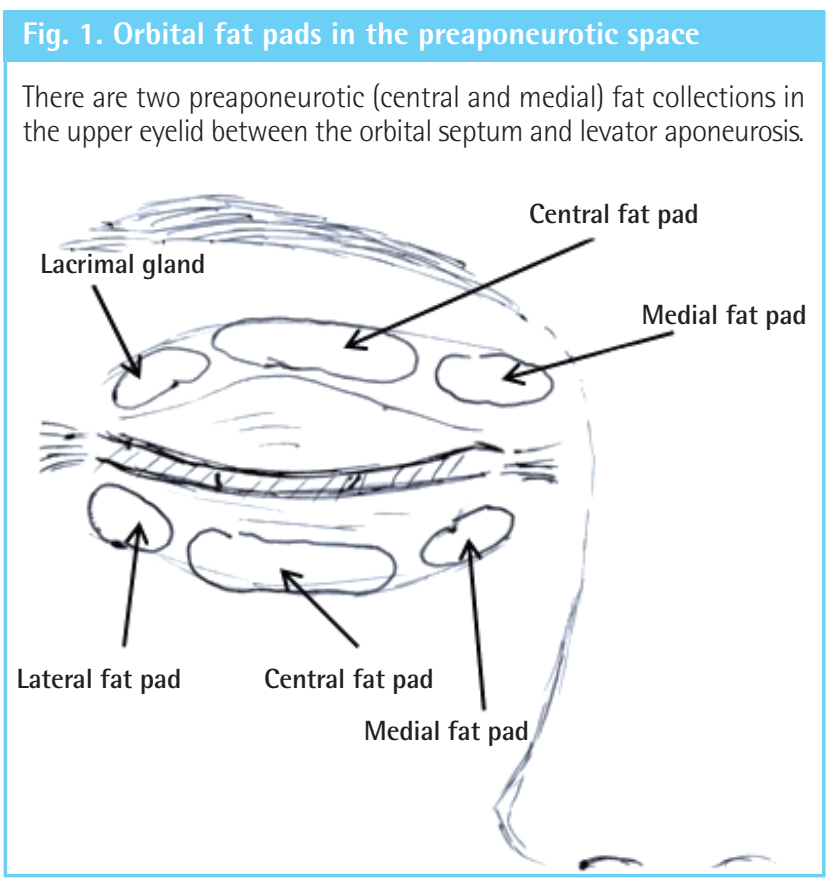

pads were identified; the yellow central fat pad was more superficial and lateral, and the white medial fat pad was deeper and medial. The lateral portion of the central fat pad was partially dissected to facilitate anchoring. The medial fat pad was gently exposed by using a pair of Metzenbaum scissors and then pulled out to facilitate anchoring. After rotating the dissected lateral portion of the central fat pad by $180^{\circ}$ to the medial side, it was anchored spreading to the medial fat pad by 6-0 Monocryl (Figs. $2,3)$. Meticulous hemostasis was carried out at every stage. Mattressed 7-0 white Nylon anchor sutures were then placed to connect the dermis of the pretarsal skin flap to the levator aponeurosis to create a supratarsal fold. The skin was repaired using simple interrupted 7-0 silk sutures. Postoperative dressing was performed on the first postoperative day, and skin sutures were removed on the fifth postoperative day.

\section{RESULTS}

All 54 patients underwent the same surgical procedure, and all the operations were performed by a single surgeon. Three of these patients were men, and the remainder were women; the mean age was 56.3 years (range, 44-64 years) for men and 54.2 years (range, 36-73 years) for women. Six patients had previously undergone an upper eyelid operation, and two patients had a history of trauma. Follow-up durations ranged from 6 to 8 months with an average of 6.2 months (Table 1). Sunken upper eyelids were effectively corrected in 51 of the 54 patients (Figs. 4,5 ). In the remaining three, the effect was minimal because preaponeurotic fat pads had been removed during previous upper blepharoplasty.

In addition to correcting sunken upper eyelids, we corrected lateral bulging and achieved improved definition of the lateral portion of the upper lid creases. No notable complications oc-

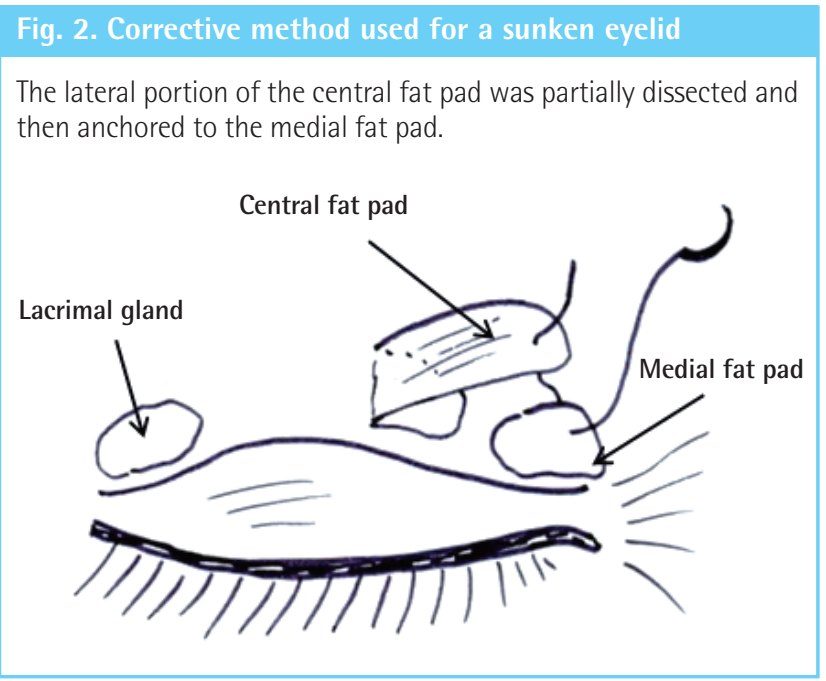




\section{Fig. 3. Intraoperative views}

(A) The lateral portion of the central fat pad was partially dissected. (B) Photograph showing the white medial fat pad. (C) The central fat pad was anchored to the medial fat pad. (D) After completion of the anchoring. Blue arrow, central fat pad; yellow arrow, medial fat pad.
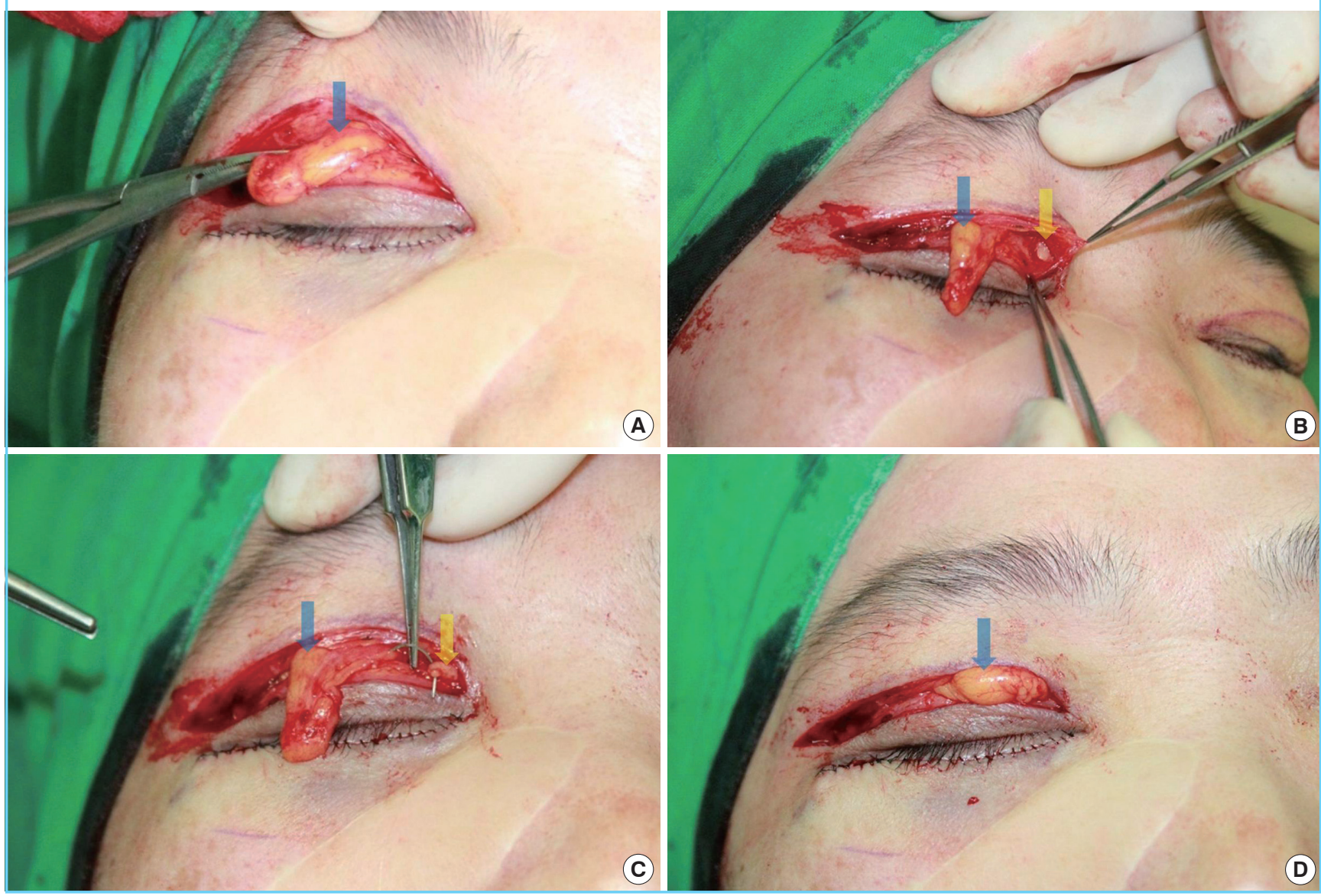

\section{Table 1. Patient demographics}

\begin{tabular}{|lc|}
\hline Characteristic & No. of patients $(\mathrm{n}=\mathbf{5 4})$ \\
\hline Sex & $3(5.6)$ \\
Male & $51(94.4)$ \\
Female & \\
Age (yr) & $44-64($ mean, 56.3) \\
Male & $36-73$ (mean, 54.2) \\
Female & $6(11.1)$ \\
Previous history of upper eyelid operation & $48(88.9)$ \\
Yes & $2(3.7)$ \\
No & $52(96.3)$ \\
Previous trauma history & $6-8$ \\
Yes & 6.2 \\
No & \\
Follow-up durations (mo) & \\
Average follow-up duration (mo) & \\
\hline Values are presented as number (\%). & \\
\hline
\end{tabular}

curred during or after surgery. Four patients underwent additional autologous fat injection because they expressed dissatisfaction with the initial results and requested further correction of sunken eyelids.
The average grading scores of the three physicians were 1.6, 1.7 , and 1.8, respectively. Cronbach's coefficient ( $\alpha$ ) for the interobserver agreement was 0.803 . The results are summarized in Table 2.

\section{DISCUSSION}

Sunken upper eyelids can be caused by normal aging or an inherited genetic predisposition [2]; for example, a strong bony orbital framework associated with delicate eyelids and soft tissue disproportion may be exhibited by several members of the same family. They can also occur after excessive fat tissue has been removed during previous blepharoplasty $[1]$ or after periorbital trauma due to a reduction in eyelid support. This is particularly the case for blowout fractures associated with a subsequent enlargement of the bony orbit and displacement or loss of orbital contents [2].

Many procedures have been used to correct sunken upper eyelids. Maniglia et al. [2] adopted surgical correction and used an orbital rim periosteal flap, which was rotated downward by $180^{\circ}$, 
Fig. 4. A 56-year-old patient

(A) Preoperative views. (B) Six-month postoperative views.
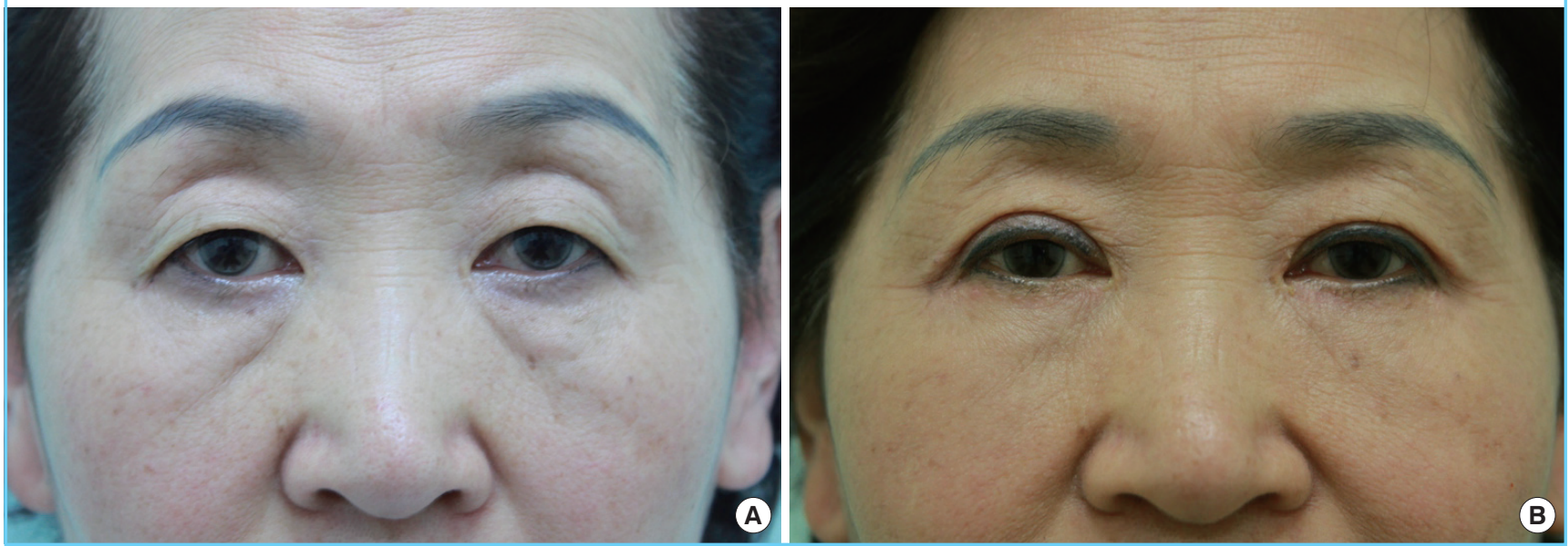

Fig. 5. A 58-year-old patient

(A) Preoperative views. (B) Six-month postoperative views.
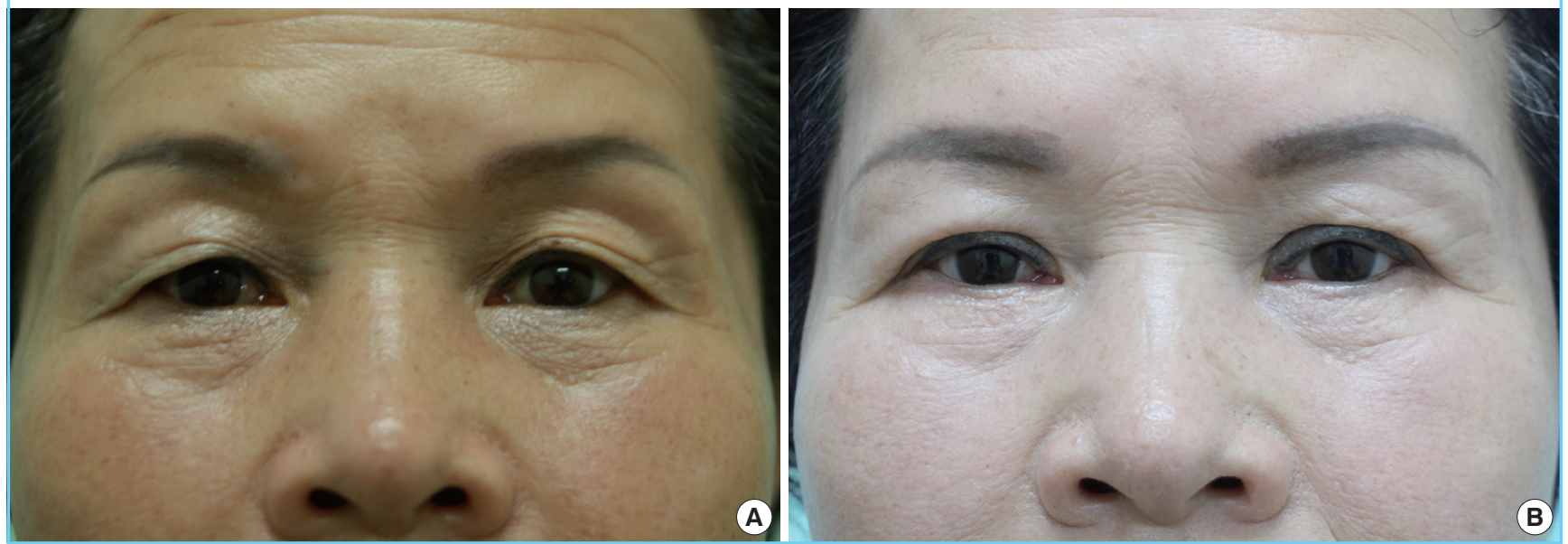

Table 2. Postoperative evaluation results of the three physicians at six-month follow-up visits

\begin{tabular}{|lccc|}
\hline \multirow{2}{*}{ Frade $^{\mathrm{b})}$} & \multicolumn{3}{c}{ Physician grading $(\mathrm{n}=\mathbf{5 4})$} \\
\cline { 2 - 4 } & First & Second & Third \\
\hline 0 & 0 & 0 & 0 \\
1 & 24 & 17 & 14 \\
2 & 28 & 34 & 35 \\
3 & 2 & 3 & 5 \\
Average grading score & 1.6 & 1.7 & 1.8 \\
\hline
\end{tabular}

a)Cronbach's coefficient $(\alpha)=0.803$; b) 0 , no correction; 1 , minimal correction; 2, moderate correction; 3 , full correction.

making a septum orbitale "curtain," and then, the released superior extraconal orbital fat was used to fill the superior periorbital space. They operated on 30 patients ( 25 bilaterally and 5 unilaterally) and achieved satisfactory results.
Autologous fat grafting is a popular method for sunken upper eyelid correction. The first use of fat in human autotransplantation was described by Van der Meulen in 1889, and a free-fat autograft in humans was first introduced by Neuber to fill a softtissue depression in 1983 [12]. Many recent reports have described various uses of autologous fat grafting, including free-fat grafts and fat injection by open and closed techniques, but these procedures have some complications including contour irregularity, fat embolism with stroke or blindness, asymmetry, infection, and fat migration [11]. Furthermore, it is difficult to predict results because fat survival depends on the individual. To overcome these problems associated with fat survival, some surgeons overcorrect sunken upper eyelids with autologous fat. However, Kim et al. [13] reported their experiences of excessive fat survival after grafting in the upper eyelid and proposed minimal autologous fat grafting in the upper eyelid to correct sunken upper eyelids 
because the grafted fat in the upper eyelids can survive more than general cases as patients gain weight. In this previous study, autologous fat grafting was performed in 15 patients with sunken upper eyelids between March 1999 and September 2006, and of the 15 patients, 4 patients with weight gain experienced excessive fat survival and required fat tissue removal to correct contour irregularities. Kim et al. [1] used dermofat grafting to correct sunken upper eyelids. Donor material was harvested between the anus and coccyx, and the fat layer of the donor material was located downwards to prevent contour irregularity. They slightly overcorrected the sunken upper eyelid by about $20 \%$. Because the postoperative absorption rate of dermofat grafting is lower than that of free-fat graft and fat injection, they could predict postoperative results more accurately and obtain satisfactory results. Lee et al. [8] used fascia-fat grafting to correct sunken upper eyelids in 13 patients with sunken and/or multiply folded upper eyelids between March 1999 and September 2006, and obtained satisfactory results.

Alloplastic materials can also be used. Morley et al. [10] used a hyaluronic acid gel for upper eyelid filling and contouring [11], Mutou [9] used a silicone bag-gel prosthesis, and Sugar and Forestner [14] used a methacrylic resin implant. However, these alloplastic materials present high risks of infection.

During surgery, after incising the skin and orbicularis muscle and dissecting the pretarsal skin and a muscle flap from the levator aponeurosis, the orbital septum was opened to expose the preaponeurotic space and the central and medial fat pads. The central fat pad was yellow, and the medial fat pad was relatively white because of their different carotenoid contents [15], and thus, they could be easily identified. The lateral portion of the central fat pad was then partially dissected to facilitate anchoring, and after rotating the dissected lateral portion of the central fat pad by $180^{\circ}$ to the medial side, it was anchored spreading to the medial fat pad. Using this technique, the medial side of a sunken eyelid was effectively corrected in most patients. However, four of the 54 patients underwent additional autologous fat injection in the subcutaneous and suborbicularis oculi layers to improve aesthetic results. We also corrected lateral bulging and improved the definition of the lateral portion of the upper lid crease by using our technique.

Our procedure has some advantages. First, it allows the correction of the medial side of the sunken upper eyelid simply and effectively during upper blepharoplasty. Second, it enables the correction of lateral bulging. Third, it provides a better-defined lateral portion of the upper lid crease. Fourth, it is free of complications such as contour irregularity, fat embolism with stroke or blindness, and donor site scarring associated with free-fat grafting and fat injection. Fifth, it allows a better prediction of postoperative results than other procedures.

Nevertheless, our procedure also has certain limitations. First, it involves an open surgical approach and is thus more aggressive than other procedures, although it is a good additional method for patients with sunken upper eyelids requesting upper blepharoplasty. Second, sunken upper eyelids are not fully corrected by using our method, particularly in the case of patients with a past history of upper blepharoplasty with the removal of fat tissue. In fact, due to small amounts of fat tissue remaining after previous surgery, only minimal effects were obtained in three of our patients. Furthermore, when our method is applied, it should be taken into consideration that the central fat pad volume diminishes more rapidly than the medial fat pad volume during aging [16]. Third, our procedure corrects sunken eyelids on the medial portion, and thus, additional procedures, such as fat grafting, are required in patients with severely sunken upper eyelids.

In conclusion, anchoring the central fat pad to the medial fat pad provides an effective means of correcting sunken upper eyelids during upper blepharoplasty but is not enough to correct sunken upper eyelids in all cases. The surgeon should consider not one method but various methods for correcting sunken upper eyelids.

\section{REFERENCES}

1. Kim YK, Lee HG. Correction of sunken upper eyelid using dermofat graft.J Korean Soc Aesthetic Plast Surg 2000;6:44-9.

2. Maniglia JJ, Maniglia RF, Jorge dos Santos MC, et al. Surgical treatment of the sunken upper eyelid. Arch Facial Plast Surg 2006;8:269-72.

3. Park S, Kim B, Shin Y. Correction of superior sulcus deformity with orbital fat anatomic repositioning and fat graft applied to retro-orbicularis oculi fat for Asian eyelids. Aesthetic Plast Surg 2011;35:162-70.

4. Jung JM, Choi WS, Jo MS. Correction of the sunken upper eyelid depending on the dermatochalasis or blepharoptosis using the autologous fat grafting. Arch Aesthetic Plast Surg 2014;20:148-54.

5. Kim YK, Lee SJ. Correction of sunken upper eyelids using autologous microfat graft. J Korean Soc Aesthetic Plast Surg 2006;12:79-82.

6. Kim SM, Jeong BR, Hong JW, et al. Correction of sunken and/or multiple folded upper eyelid by refined structural micro-fat graft: technical pearls. Arch Aesthetic Plast Surg 2013;19:29-33.

7. Kwon SM, Park J, Yang WY, et al. Correction of sunken eyelid with unfavorable fold using autologous fat injection. J Ko- 
rean Soc Plast Reconstr Surg 2008;35:471-9.

8. Lee Y, Kwon S, Hwang K. Correction of sunken and/or multiply folded upper eyelid by fascia-fat graft. Plast Reconstr Surg 2001;107:15-9.

9. Mutou Y. Use of a silicone bag-gel prosthesis to fill in a supratarsal depression of the upper eyelid. Plast Reconstr Surg 1978;62:862-5.

10. Morley AM, Taban M, Malhotra R, et al. Use of hyaluronic Acid gel for upper eyelid filling and contouring. Ophthal Plast Reconstr Surg 2009;25:440-4.

11. Collar RM, Boahene KD, Byrne PJ. Adjunctive fat grafting to the upper lid and brow. Clin Plast Surg 2013;40:191-9.

12. Billings E Jr, May JW Jr. Historical review and present status of free fat graft autotransplantation in plastic and reconstructive surgery. Plast Reconstr Surg 1989;83:368-81.

13. Kim DK, Hong SH, Hong SE. Experiences in excessive fat survival after fat graft in upper eyelid.J Korean Soc Aesthetic Plast Surg 2009; 15:82-4.

14. Sugar HS, Forestner HJ. Methacrylic resin implant for sunken upper lid following enucleation. Ill Med J 1947;92:219-24.

15. Sires BS, Saari JC, Garwin GG, et al. The color difference in orbital fat. Arch Ophthalmol 2001;119:868-71.

16. Oh SR, Chokthaweesak W, Annunziata CC, et al. Analysis of eyelid fat pad changes with aging. Ophthal Plast Reconstr Surg 2011;27:348-51. 